\title{
VEJEZ Y SOLEDAD: IMPLICANCIAS A PARTIR DE LA CONSTRUCCIÓN DE LA NOCIÓN DE RIESGO
}

Jean Gajardo Jauregui ${ }^{1}$

Resumen: El envejecimiento de la población supone desafíos particulares en la búsqueda de bienestar de las personas mayores. La soledad es un fenómeno de creciente relevancia en la investigación y las políticas públicas relacionadas con vejez y envejecimiento, debido a su relación con una etapa de la vida asociable a pérdidas sociales y a su influencia en la salud mental de la persona mayor. Este ensayo busca reflexionar sobre la soledad situada en la vejez desde diferentes racionalidades del concepto, como categoría de clasificación cultural y epidemiológica y las implicancias que esta puede tener en la definición de políticas y estrategias para su abordaje. Se enfatiza en la atingencia de una racionalidad de la soledad a partir de la construcción cultural del riesgo y subjetivación del daño, por sobre la noción medicalizada del fenómeno como síndrome o factor de riesgo epidemiológico. Finalmente, se propone la consideración de escenarios de construcción cultural para la racionalidad de la soledad en la vejez y se discute sobre consideraciones para su medición y su abordaje en el marco de una racionalidad enmarcada en una epidemiología cultural.

Palabras clave: soledad, vejez, envejecimiento, riesgo, cultura

\section{Aging and loneliness: implications from building the notion of risk}

\begin{abstract}
Population aging implies specific challenging when looking for the wellbeing of elderly. Loneliness is an issue of increase relevance in research and public policies related to old age and aging, due to its relation to a life stage associated to social losses and is influence in the mental health of the elderly. This essay tries to reflect about loneliness in old age from different rationalities of the concept, as category of cultural and epidemiological classification and the implication it may have in the definition of public policies and strategies to approach the issue. The relation to a rationality of loneliness built from the culture of risk and damage subjectivity is emphasized over the medicalization of the issue as disorder or epidemiological risk factor. Finally, the consideration of building cultural stages to rationalize loneliness in old age is proposed and considerations to measure and approach the issue in the rational framework of cultural epidemiology are discussed.
\end{abstract}

Key words: loneliness, old age, aging, risk, culture

\section{Velhice e solidáo: implicaçóes a partir da construçáo da noçáo de risco}

Resumo: O envelhecimento da população supóe desafios particulares na busca de bem-estar das pessoas idosas. A solidão é um fenômeno de crescente relevância na investigação e nas políticas públicas relacionadas com a velhice e o envelhecimento, devido à sua relaçáo com uma etapa da vida associada a perdas sociais e à sua influência na saúde mental da pessoa idosa. Este ensaio busca refletir sobre a solidão situada na velhice a partir de diferentes racionalidades do conceito, como categoria de classificação cultural e epidemiológica e as implicaçôes que esta pode ter na definição de políticas e estratégias para sua abordagem. É enfatizada a obtenção de uma racionalidade da solidão a partir da construção cultural do risco e subjetivação do dano, sobre a noção medicalizada do fenômeno como síndrome ou fator de risco epidemiológico. Finalmente, propõe-se a consideração de cenários de construção cultural para a racionalidade da solidão na velhice e discute-se sobre consideraçóes para sua medição e sua abordagem no marco de uma racionalidade enquadada numa epidemiologia cultural.

Palavras-chave: solidão, velhice, envelhecimento, risco, cultura

\footnotetext{
${ }^{1}$ Escuela de Salud Pública, Universidad de Chile, Chile Correspondencia: jgajardo@med.uchile.cl
} 


\section{Introducción}

El envejecimiento de la población, con los matices particulares en cada país o región, es un fenómeno global definido por la Organización de las $\mathrm{Na}$ ciones Unidas como un proceso sin precedentes y que se mantendrá en aumento, proyectándose la duplicación del número de personas de 60 años o más en 2050, con un número estimado de 21 mil millones en el mundo(1). América Latina y el Caribe han experimentado un ritmo acelerado de envejecimiento(2), con características propias en la experiencia individual y social de envejecer según las características culturales de la región(3). Se estima que la proporción de población de 60 años y más aumentará desde un $8 \%$ en 2000 a $14,1 \%$ en 2025 y a $22,6 \%$ en 2050 , cuyo impacto no solo dice relación con su expresión cuantitativa sino con el escenario social y económico en el que aún se encuentra la mayor parte de los países de esta área, caracterizada por inequidad y baja cobertura de la seguridad social(4).

En Chile, según las proyecciones del CENSO 2002, las personas de 60 años y más representaban en 2010 el 13\% de la población total y se estima que este porcentaje continuará aumentando durante esta década llegando al 20\% en 2025(5). El envejecimiento poblacional plantea diversos desafíos en su comprensión y en los conceptos que involucre, lo que tiene una implicancia práctica en la generación de políticas públicas para la promoción del bienestar de las personas adultas mayores.

Si bien el envejecimiento y la vejez son fenómenos altamente heterogéneos, al relevarlos en sus dimensiones culturales emerge la idea de la existencia de envejecimientos normativos, que se enmarcarían como formas, mecanismos o procesos que se naturalizan y cuya extensión y reproducción los convierte en norma social(6). A partir de esto, sería posible reconocer formas de envejecer que posean una mayor deseabilidad social y que, por tanto, se configurarían como objetivos de la gestión política de un país y, de manera consecuente, otras formas de envejecimiento no normativas que se alejen de dicha norma social naturalizada o esperada. Se ha propuesto que las personas que no envejecen "como suelen hacerlo los demás" se adaptan a las estructuras disponi- bles para organizar tanto su cuidado en la vejez como en otras esferas de su vida(6), por lo que un insumo relevante en la planificación de servicios y de política pública que apoye a estos grupos debe considerar el análisis reflexivo sobre las racionalidades y conceptos en los que se apoye. Estudios sobre envejecimientos no normativos han sido desarrollados, por ejemplo, en adultos medios y adultos mayores que no han tenido hijos, personas mayores homosexuales, bisexuales y transgénero, personas que envejecen en contextos de hábitos religiosos, personas mayores migrantes y personas mayores en situación de calle.

A partir del estudio de estas realidades, se ha mencionado un elemento de interés como condición vinculada a algunos de los envejecimientos previamente referidos: la situación de soledad en la vejez. ¿Qué se entiende por una vejez en soledad y por qué suele relacionarse con riesgo? ¿Qué racionalidades pueden describirse para el concepto y la construcción de riesgo asociado a este?

Mediante este manuscrito se buscará reflexionar sobre el concepto de soledad en la vejez, desde sus diferentes racionalidades como categoría de clasificación cultural y epidemiológica y las implicancias que estas puede tener en la definición de políticas y estrategias para su abordaje.

\section{Racionalidades en la soledad y su noción como factor de riesgo}

En Chile, en 2012, el Servicio Nacional del Adulto Mayor elaboró la Política Integral de Envejecimiento Positivo, con el propósito de delinear las acciones concernientes a un buen envejecimiento y vejez en Chile. Dicha política busca enfrentar el desafío del envejecimiento y la vejez en toda su magnitud, partiendo por el Estado, en conjunto con la sociedad civil y las familias chilenas. La política declara en su marco teórico que la soledad es un factor de riesgo, especialmente cuando está asociada a estereotipos negativos(7).

En 2009 se estimó que en Chile un 85\% de los adultos mayores vive en zona urbana, $16 \%$ de las personas adultas mayores viven solas, un $35 \%$ vive con su familia sin cónyuge y un $47 \%$ vive con su cónyuge y familia. Sobre el $80 \%$ de los cuidados a personas mayores dependientes son 
realizados por mujeres, principalmente hijas y cónyuges(8). Se ha mencionado también que los hombres mayores tienen niveles de satisfacción con su vida superior al de las mujeres mayores(9). Dichos antecedentes informan a grandes rasgos sobre la realidad de la conformación social de las personas mayores y permiten reflexiones iniciales sobre las implicancias de género y la configuración de protección y cuidado social en la vejez.

La soledad puede ocurrir en cualquier edad y, si bien la vejez no es la responsable directa o única en la generación de situaciones de soledad en personas mayores, se ha descrito una relación según eventos frecuentes en esta edad, tales como el fallecimiento del cónyuge y de otros componentes de la red social(10). La soledad en la vejez se ha asociado con impactos individuales, como depresión y aislamiento social, y la información sobre sus causas es controversial(11). De acuerdo con los datos previamente mencionados, ¿podría afirmarse que el $16 \%$ de personas mayores que viven solas en Chile se encuentran en riesgo?

Dicho planteamiento resulta relevante en el marco del concepto - creencia- de un envejecimiento positivo, en el que se reconoce la soledad como un elemento de riesgo. La racionalidad de la soledad en la vejez como un factor de riesgo se ha desarrollado principalmente a partir de la investigación epidemiológica, al notar su asociación con otras circunstancias patológicas, mayormente de índole psicológico(12). La soledad, desde esta perspectiva, encuentra su racionalidad en la medicalización de su noción, como un síndrome que puede ser prevenido, detectado y tratado(13), y el análisis detrás de su medición encuentra lugar por medio del establecimiento de una definición numérica o categórica del estado (por ejemplo, considerando la situación de soledad como la ausencia de cónyuge, o una red social reducida de acuerdo a algún estándar). Luego, dicha racionalidad se enfocaría en el riesgo individual a partir de medir la soledad como un fenómeno objetivo y externo que permite la generación de categorías de personas según su riesgo.

Ahora bien, la categoría de factor de riesgo o síndrome tiene una implicancia no solo como discurso y análisis, sino que también en la determinación de acciones relacionadas con el problema.
En una revisión sistemática realizada en 2011, se evaluaron los resultados de diversas intervenciones para el aislamiento social y la soledad en adultos mayores(14). Una primera conclusión relevante, a partir de dicha investigación, fue la existencia de alta heterogeneidad de intervenciones para la reducción de la soledad que fueron encontradas y analizadas. Los autores reflexionan sobre la diversidad en torno a la conceptualización de la soledad y las implicancias que esto tiene en el planteamiento de acciones. Es interesante observar que las acciones dirigidas a la soledad se enfocan únicamente en las personas adultas mayores en soledad e incorporan diversas acciones centradas en la asistencia social y el apoyo psicológico, en un formato de entrega de servicios con temporalidad transversal y centrados en el individuo (aunque utilice metodologías grupales), con escasa mención a las circunstancias biográficas o culturales en las que se encuentran las personas. Luego, podría interpretarse que las intervenciones hacia la soledad se desarrollan desde la medicalización del concepto, según la racionalidad que ha sido descrita.

Dicha racionalidad puede ser analizada también a partir de la producción de conocimiento sobre el riesgo y la idea de reducción del daño(15). ¿Cómo interactúan el riesgo y la vulnerabilidad en este grupo? No es fácil responder a estas preguntas bajo una única racionalidad epidemiológica sobre el fenómeno, en pos de medir un fenómeno externo, estático y atemporal, en el intento por generalizar un problema que desde la estadística puede percibirse como tal, pero no necesariamente para los sujetos.

Frente a esto, se describe la idea del riesgo como una construcción social que requiere la inclusión de representaciones y percepciones frente al daño, con el propósito de relativizar las nociones de riesgo epidemiológicas(16) y una mirada a la construcción del riesgo epidemiológico desde una visión más cercana a la hermenéutica que al determinismo de los fenómenos que se intenten describir(17). Luego, el estudio del riesgo presente en la situación de soledad en la vejez recibe aportes desde la antropología a partir de una mejor comprensión del proceso y los condicionantes que están implicados. Al respecto, un elemento fundamental que es también referido por Trostle 
es el estudio de la representación: ¿qué representación elaboran los sujetos en torno a la soledad? ¿Incide esta en la construcción del riesgo? ¿Es la soledad siempre una condición negativa desde la percepción de las personas? ¿Están de algún modo involucradas las representaciones sobre un envejecimiento y vejez positivos en la construcción de este fenómeno como un factor de riesgo que proteja de un envejecimiento y una vejez negativos? ¿Cómo participan los contextos de imaginarios y expectativas sociales respecto de nuestra protección social?

El desarrollo del concepto de "soledad" ha puesto también en diálogo una construcción del riesgo a partir de la experiencia y representaciones mismas de los sujetos, orientándose a la idea de vulnerabilidad centrada en las interacciones de los actores y contextos involucrados en el fenómeno, por sobre una idea de riesgo delimitable desde una mirada epidemiológica. Dicha construcción de vulnerabilidad se genera de forma coherente con la biografía y las creencias, y da cuenta de mejor forma de una construcción cultural de riesgo. Luego, el concepto de "soledad" es replanteado desde su neutralidad y estaticidad original hacia un concepto dinámico y más permeable. A partir de esto, la conceptualización y medición del fenómeno no se centra en el ideal de una red social, sino en cómo el sujeto perciba su propia realidad social, sea cual sea la conformación objetiva de esta. En relación con lo planteado, se ha descrito la evolución de la noción de riesgo como cultural, pasando de explicación de fenómenos desde la naturalización hacia enfoques más interpretativos y de construcción(18). Según dicho planteamiento, este abordaje permite reducir la disonancia entre la percepción de las personas y la percepción de los expertos, y favorece una mejor medición e identificación de los sujetos en riesgo.

De este modo, entra en juego la conceptualización y medición de la soledad a partir del complemento entre la antropología y la epidemiología. Se ha planteado que las miradas epidemiológica y antropológica no representa polos en un mismo espectro metodológico, sino que acuerdos destinados a producir con mayor eficiencia conocimientos relacionados con problemas, hacia una etnoepidemiología(19). Dicha aproximación también aporta en la consideración de los con- textos sociales y de representación sobre nuestros vínculos sociales y las experiencias sociales de las personas adultas mayores en sus realidades. De este modo, la complementariedad entre epidemiología y antropología ofrecería el potencial de conceptualizar y medir la soledad en la vejez en forma más adecuada, y la posibilidad de una deconstrucción enmarcada del concepto en adecuación al tiempo, espacio y representaciones sociales.

Es así como el concepto de "soledad" puede complejizarse a partir del reconocimiento de la valoración del propio sujeto en relación con su percepción de la compañía social que posea, por ejemplo, a partir de mediciones que se centren en el sentirse solo/a por sobre el estar solo/a, lo que entrega un marco de generación de intervenciones centrado en la facilitación de establecimiento de vínculos sociales y no en la suplencia o entrega deliberada de acompañamiento desde una mirada biomédica del problema(20).

Si se enfatiza en la idea de una construcción del riesgo y la vulnerabilidad, la soledad puede ser descrita como una sensación de aislamiento de otras personas, sin importar si se está aislado físicamente de los demás o no, y muchas personas tienen momentos en los que están solos, por circunstancias o elección, lo que puede ser experimentado como algo positivo, placentero, y emocionalmente refrescante si está bajo el control del individuo $(21,22)$. La soledad en la vejez responde luego a una condición de estado emocional que se produce cuando una persona se siente distanciada de, o incomprendido o rechazado por otros, y/o carece de los recursos sociales adecuados para llevar a cabo las actividades que desee, particularmente las actividades que proporcionan un sentido de integración social y las oportunidades para la intimidad emocional con otras personas $(23,24)$.

Por medio de aproximaciones cualitativas se ha logrado conocer, por ejemplo, que la soledad se relaciona con procesos de producción en los que el adulto mayor ha experimentado ausencia o quiebre de relaciones importantes en su historia personal, entre los que destacan: soledad como respuesta al dolor y duelo que acompaña el término de relaciones con seres queridos, el intento de 
evitación o estrategias de afrontamiento al dolor que no son útiles, la relación con temor, tristeza y ansiedad por miedo a la discapacidad y dependencia, y como estado de sufrimiento en el que la persona se niega a sí misma o es incapaz de verbalizar sus necesidades de compañía(25). De este modo, la conceptualización del problema y las aproximaciones a su medición toman otro marco de racionalidad, enfatizando en la valoración de la experiencia y la subjetivación del daño.

A partir de lo descrito, desde una racionalidad de la soledad que se oriente a la construcción cultural del riesgo desde una visión etnoepidemiológica, la exploración del fenómeno puede considerar escenarios de interacción para su análisis que orienten de este modo su conceptualización, medición y abordaje. A continuación (figura 1) se propone una forma de conceptualizar los escenarios de interacción a considerar en la elaboración de racionalidades sobre la soledad, los que se basan primariamente en la idea cultural sobre la compañía y la presencia social de la persona que envejece, y considerando la premisa de una influencia dinámica entre el individuo y su entorno.

Desde estas perspectivas, cabe preguntarse, en el contexto de nuestro país, ¿qué sucede con la integración social de las personas adultas mayores? ¿Cómo significamos culturalmente su rol y cómo posibilitamos las oportunidades de participación social? ¿Podríamos asegurar, en nuestro contexto, que si una política de envejecimiento positivo aborda la reducción de la soledad, dichos elementos estructurales se encuentran previamente satisfechos y, por ende, se estaría interviniendo en efecto el fenómeno aludido?

\section{Conclusiones}

La soledad en la vejez es un concepto complejo que encuentra distintas racionalidades en su comprensión, las que suponen diferentes consideraciones para su medición y abordaje. Por una parte, es posible encontrar una noción medicalizada del fenómeno, describiéndolo como un síndrome o un factor de riesgo detectable y prevenible desde la epidemiología. Dicha noción se relaciona con una homogenización de un riesgo individual atribuible a partir de la objetivación del fenómeno. De manera consecuente, la idea del abordaje se relaciona con la intervención para su reducción.

Por otra parte, desde una aproximación etnoepidemiológica o de epidemiología cultural es posible analizar el fenómeno de la soledad a partir de una construcción de riesgo, considerando la heterogeneidad de experiencias y representaciones de envejecimiento y vejez en compañía, enfatizando en la idea de vulnerabilidad a partir

Figura 1: Escenarios de construcción cultural para la racionalidad de la soledad en la vejez.

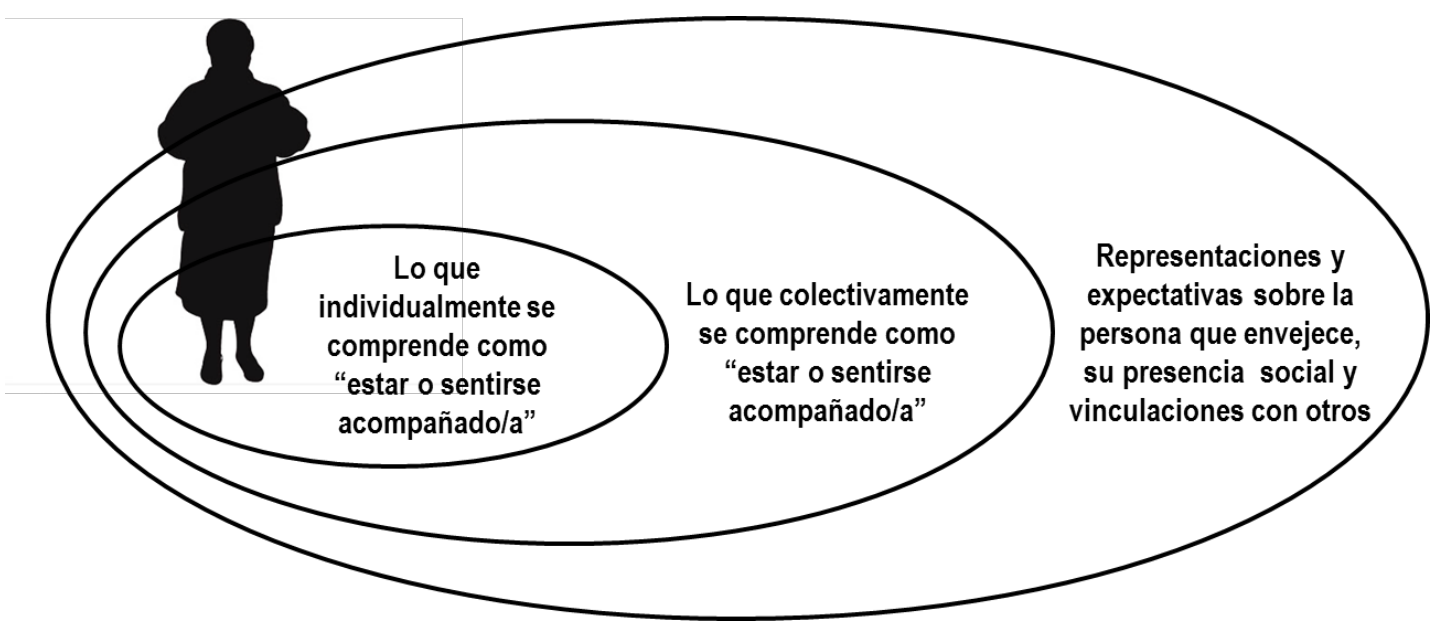

Fuente: Elaboración propia. 
de las interacciones que las personas tienen en un medio cultural, por sobre la identificación de un riesgo. De este modo, la medición y acciones hacia el fenómeno pueden considerar con mayor dinamismo la relación que las personas que envejecen establecen entre sus propias expectativas y su evaluación de red social o de compañía, con el contexto cultural colectivo sobre lo que se represente como una vejez en compañía y la presencia social que la vejez tenga en su medio cultural en términos de oportunidades y expectativas para la vinculación social.

A partir de lo anterior, puede observarse que en sociedades como la chilena, que disponen de un modelo de familia que se centra en la relación conyugal heterosexual como su núcleo base y un modelo centrado en la capitalización individual por sobre un Estado de Bienestar, la familia se constituye como la principal forma de acceso a compañía y protección social de las personas. Esta situación puede relacionarse luego con la expectativa social de un cierto envejecimiento y una vejez en compañía como una situación normalizada, y como base para el sostén de cuidados y bienestar, lo que constituiría el escenario cultural colectivo en el cual los sujetos evalúan y contrastan su propia experiencia de compañía o soledad.
Bajo dicha consideración, el abordaje de la soledad por medio de políticas públicas debiese abordar primariamente los escenarios culturales y de representaciones, promoviendo visiones positivas de envejecimientos diversos y buscando la protección social de la persona mayor de acuerdo con sus propias expectativas culturales, brindando los apoyos asistenciales necesarios de acuerdo con la cultura y biografía de las personas. Las personas adultas mayores son sujetos con una historia y que han construido su vinculación con el entorno a lo largo de su experiencia vital y, por ende, es necesario incluir estrategias que consideren el curso de vida por sobre intervenciones específicas en el grupo etario. ¿De qué forma en Chile se promueve la vinculación y la protección social de las personas reconociendo la diversidad de potenciales formas que existen en la realidad, distintas a la constitución de matrimonio civil y familia nuclear?

Finalmente, la racionalidad y desarrollo de acciones hacia la soledad en la vejez debiese acompañarse de la observación crítica sobre el modo en que se promueve la participación de las personas mayores, en un escenario cultural que tiende a mirar la vejez de forma proteccionista y pesimista (26-28). 


\section{Referencias}

1. Organización de las Naciones Unidas. World Population Ageing 1950-2050: Resumen ejecutivo; 2002. Available from: http://www.un.org/esa/population/publications/worldageing19502050/

2. Palloni A, Pinto-Aguirre G, Pelaez M. Demographic and health conditions of ageing in Latin America and the Caribbean. Int J Epidemiol 2002; 31(4): 762-771.

3. George L. Prepared or Not. Latin America Faces the Challenge of Aging 2011; 75-80.

4. Guzmán JM. Envejecimiento y desarrollo en América Latina y el Caribe. Santiago; 2002. Available from: http://www.eclac. org/publicaciones/xml/4/10364/serie28.pdf

5. Instituto Nacional de Estadísticas. Chile: Proyecciones y estimaciones de población. Total país 1950-2050; 2004. Available from: http://www.ine.cl/canales/chile_estadistico/demografia_y_vitales/proyecciones/Informes/Microsoft Word -InforP_T.pdf

6. Zamora G. Prestación de cuidados y envejecimiento en personas sin hijos. Actas de la Dependencia 2013; 8: 1-15.

7. Servicio Nacional del Adulto Mayor, Chile. Política Integral de Envejecimiento Positivo 2012 - 2025; 2012. Available from: http://www.senama.cl/filesapp/PoliticaAM.pdf

8. Servicio Nacional del Adulto Mayor, Chile. Estudio nacional de la dependencia en personas mayores; 2009.

9. Pontificia Universidad Católica de Chile. Chile y sus mayores: Resultados Segunda Encuesta Nacional Calidad de Vida en la Vejez; 2010. Available from: http://www.senama.cl/filesapp/ChileYsusMayores_EncuestaCalidaddeVida.pdf

10. Hazer O, Boylu AA. The examination of the factors affecting the feeling of loneliness of the elderly. Procedia - Soc Behav Sci 2010; 9: 2083-2089.

11. Cacioppo JT, Hawkley LC, Ernst JM, Burleson M, Berntson GG, Nouriani B, et al. Loneliness within a nomological net: An evolutionary perspective. J Res Pers 2006; 40(6): 1054-1085.

12. Grenade L, Boldy D. Social isolation and loneliness among older people: issues and future challenges in community and residential settings. Aust Health Rev 2008; 32(3): 468-478.

13. Rodríguez M. La soledad en el anciano. Gerokomos 2009; 20(5): 159-166.

14. Dickens AP, Richards SH, Greaves CJ, Campbell JL. Interventions targeting social isolation in older people: a systematic review. BMC Public Health 2011; 11(1): 647.

15. Nitcher M. Reducción del daño: una preocupación central para la antropología médica. Desacatos 2006; 20: 109-132.

16. Trostle J. Perceiving and representing risk. Epidemiology and Culture. 1st ed. Cambridge University Press; 2005: 150-161.

17. De Almeida N, Castiel LD, Ayres JR. Riesgo: concepto básico de la epidemiología Salud Colect 2009; 5(3): 323-344.

18. Gabe J, Bury M, Elston A. Risk. Key Concepts in Medical Sociology. $1^{\text {st }}$ ed. SAGE Publications; 2004: 87-91.

19. De Almeida N. Por una epidemiología con (más que) números: cómo superar la falsa oposición cuantitativo-cualitativo. Salud Colect 2007; 3(3): 229-233.

20. Ekwall AK, Sivberg B, Hallberg IR. Loneliness as a predictor of quality of life among older caregivers. J Adv Nurs 2005; 49(1): 23-32.

21. Berg S, Mellstrom D, Persson G, Svanborg A. Loneliness in the Swedish Aged. J Gerontol 1981; 36(3): 342-349.

22. Andersson L. Loneliness research and interventions: A review of the literature. Aging Ment Health 1998; 2(4): $264-274$.

23. Rook KS. Promoting Social Bonding Strategies for Helping the Lonely and Socially Isolated. Am Psychol 1984; 39(12): 1389-1407.

24. Donaldson JM, Watson R. Loneliness in elderly people: an important area for nursing research. J Adv Nurs 1996; 24(5): 952-959.

25. McInnis GJ, White JH. A phenomenological exploration of loneliness in the older adult. Arch Psychiatr Nurs 2001; 15(3): 128-139.

26. North MS, Fiske ST. Subtyping Ageism: Policy Issues in Succession and Consumption. Soc Issues Policy Rev 2013; 7(1): 36-57.

27. Arnold-Cathalifaud M, Thumala D, Urquiza A, Ojeda A. El imaginario de los jóvenes sobre la vejez en Chile: estudio exploratorio. Available from: http://www.facso.uchile.cl/observa/04.pdf

28. Toledo AM. Viejismo. Percepciones de la población acerca de la tercera edad: estereotipos, actitudes e impliancias sociales. Rev Electron Psicol Soc. 2010; 19(1):1-10.

Recibido: 2 de septiembre de 2014

Aceptado: 12 de noviembre de 2014 